\title{
Wireless Echocardiographic Image Acquisition and Review
}

\author{
NL Greenberg, G Saracino, AG Borowski, JD Thomas \\ The Cleveland Clinic Foundation, Cleveland, USA
}

\begin{abstract}
Digital echocardiography has altered clinical workflow and improved patient care. While network performance is critical for data transfers, bandwidth tradeoffs might be acceptable to allow access to data outside of the echo laboratory. In this study, we examined the ability to use portable ultrasound in a wireless environment to provide real-time echo monitoring. Video output of a handheld machine transmitted over a wireless infrastructure in which various parameters were modulated to assess their impact. In this testbed configuration, we are able to transmit full color echocardiographic images via $802.11 \mathrm{~b}$ to a handheld computer with a resolution of $320 \times 240$ pixels at 15 fps. While a major strength of ultrasound is its portability, wired network connectivity creates limitations on effective data management. The growth of handheld echocardiographic devices further emphasizes the need for wireless connectivity beyond the boundaries of the hospital.
\end{abstract}

\section{Introduction}

Digital echocardiography has dramatically impacted the ability for diagnostic interpretation of echocardiograms. The ability to access centralized DICOM data for data review and interpretation is a useful capability that can lead to more efficient utilization of physician time, easier access to advanced expertise, and more rapid interpretation of patient data.[1][2] Network performance is critical to move image data effectively, but bandwidth tradeoffs may be acceptable to provide access to data outside the echo laboratory. We have extended our laboratory's DICOM network to include five satellite facilities up to $100 \mathrm{~km}$ from the main campus using T1 connections. Together, these sites generate approximately 20 echocardiographic studies per day requiring transfer of around $1 \mathrm{~GB}$ of echo data to our archive over T1 and T3 lines. This is in addition to the 200 studies and $20 \mathrm{~GB}$ data generated on the main campus itself. Access to data has also been extended to physician homes using cable modem access with a secure VPN (virtual private networking) protocol, allowing for secure, encrypted transfer of data.

While wired ultrasound device connectivity is required today, wireless technologies will be introduced soon to allow data to be moved more easily throughout the hospital. To explore the feasibility of acquiring and reading echocardiograms in a wireless fashion, we hypothesized that a small ultrasound system could be utilized within a wireless environment to provide truly portable real-time echo monitoring.

There are several convergent trends in medicine and technology that can be integrated to improve health care. Among these convergent trends are the following: (1) ultrasound has emerged as the most commonly applied diagnostic imaging test; (2) miniaturization has led to high quality, light weight ultrasound imaging equipment; (3) internationally standards for digital file storage in ultrasonography; (4) growth of the internet and other networked applications has allowed remote telemedicine using ultrasound; (5) explosion in wireless telemetry and bandwidth should allow telemedicine applications in remote areas underserved by hardwired networks; (6) ultrasound is now routinely used to guide therapeutic interventions, with further automation possible for remote guidance; (7) as ultrasound technology advances, realtime three-dimensional ultrasonography will emerge as a standard of acquisition which should simplify the problem of poorly trained individuals acquiring images of diagnostic quality.

\section{Methods}

\subsection{Portable ultrasound systems}

While several vendors have introduced small ultrasound systems, relatively few data exist regarding their accuracy in a variety of medical disorders. Furthermore, there are scarcely any data comparing these systems. In a field as rapidly developing as this one, it is critical to develop metrics of usability and accuracy so that the proper choice in instrument can be made for a particular application.

To date we have examined the following systems: SonoHeart (SonoSite, Bothell, WA); Optigo (Philips, Andover, MA); Cypress (Siemens, Mountain View, CA). These miniature echo machines have been surveyed for such features as 1) weight; 2) battery life; 3) boot-up time; 4) screen luminosity and visibility under a variety of 
lighting conditions; 5) available imaging modalities (2D imaging, color flow Doppler, pulsed wave Doppler, continuous wave Doppler, M-mode with and without color Doppler); 6) completeness of measurement package; 7) output capabilities (analog video, digital still and movie export, adherence to the DICOM standard); 8) image resolution from a standard ultrasound phantom. Experimental testing to assess their diagnostic capabilities has also been initiated. For cardiac assessment, patients scheduled for a clinical echocardiographic study have participated, undergoing both a standard imaging exam as well as one with a portable system. The SonoHeart portable handheld ultrasound instrument (Sonosite, Bothell, WA) was utilized in this study to acquire cardiac images.

\subsection{Wireless test bed}

Many currently available wireless technologies including Bluetooth, 802.11a, 802.11b, and 802.11g were evaluated for transfer of ultrasound data. The $802.11 \mathrm{~b}$ standard is ideal for deployment of low bit-rate video streams over an expanded range. Nominally $802.11 \mathrm{~b}$ is able to transfer data wirelessly up $430 \mathrm{ft}$ from the access point at a reasonable speed of 2 Mbps. Mixing the 802.11.b standards and 802.11.g standards has a negative impact over the overall responsiveness of the dual standard router due to the different protocols that the router has to handle.

A wireless test bed was implemented within the laboratory. This wireless network enables us to transfer within the hospital and remotely to workstations connected to our network through a Virtual Private Network (VPN) protocol. The testbed includes wireless connection to desktop PC (802.11g 54Mbits with maxrange: $75-150 \mathrm{ft}$ ), wireless connection to handheld PC $(802.11 \mathrm{~b}$ within range $75-150 \mathrm{ft}$ ), wired connection to local PC (TCP/IP 100 mbits) and wired connection to remote PC through VPN. We chose to base the test infrastructure on the standard $802.11 \mathrm{~g}$ because, not only does it offer the same speed of 802.11a at a fraction of the cost, but also it enables integration the inexpensive 802.11.b standard available even for the Windows CE platform.

\subsection{Video encoding}

The video output from the SonoHeart scanner was digitized and encoded on a laptop PC. The acquisition board currently used is the Hauppauge PC/TV pro. Since it is USB powered, this solution enabled us to perform an entirely wirelessly acquired echocardiographic examination. Among the video streaming software solutions evaluated were: Microsoft Media Encoder, Microsoft Media Server, and Microsoft Media Player for Windows and Pocket PC. This solution has the advantages that video can be easily integrated within web pages (multi-platform visualization), Windows media player is included in Win CE 3.0 (no extra software needed), and Windows Media Server License is included with Windows 2000 Professional Server Edition.

The encoded video stream was stored to a web server for real-time review or playback at a later time. A preliminary study of 5 patients was conducted in which video encoding codecs (WM7, WM8, DivX 5, and Microsoft MP4) and transmission rates (150 to 2000 kbits) were varied.

\section{Results}

\subsection{Performance}

In this testbed configuration, we are able to transmit full color echocardiographic images via $802.11 \mathrm{~b}$ to a handheld computer with a resolution of $320 \times 240$ pixels at 15 fps. While current technologies limit the extent of high bandwidth wireless networking, emerging wireless standards (e.g., 802.11g) may permit real-time review of full resolution echos by a remote expert.

In our laboratory testbed configuration, our physicians were successfully able to read and interpret examinations acquired and transmitted at VHS quality (320x200@ 30fps). The minimum requirement for such acquisition was a PII $366 \mathrm{MHz}$ with $256 \mathrm{MB}$ Ram. Our tests showed the ideal encoding standard is currently Microsoft MP4 at $600 \mathrm{Kbit}$ with key-frame generated every 5 seconds. We conducted a preliminary study of 5 patients imaged using a portable device. Images were encoded using windows media encoder (VHS quality 320x200@30fps) using different codecs including WM7, WM8, DivX 5, and Microsoft MP4. The transmission bit rate ranged from 150 to 2000 kbits during wireless transfer of streaming video to a Compaq HP3800.

\subsection{Handheld portable review}

We obtained successful visualization on Pocket PC using Microsoft Media Player with maximal bit rates < 1500 kbits. However, the Windows CE 3.0 handheld (Compaq HP 3800) was not able to correctly process the video stream at a frame rate of $30 \mathrm{fps}$. Even at a low bit rate, streaming resulted in frequent interruption (freezing) of full motion video, which resulted in difficult diagnostic reading on the WinCE device. On the other hand, visualizations of the same streams were flawless, in all tests, on a wirelessly connected desktop computer running Windows 2000 Professional. Therefore, we can conclude that our system was capable of successfully sending wireless VHS quality video streams.

The WinCE handheld was able to flawlessly play video content with max frame rate of $15 \mathrm{fps}$. We identified the limitation in the video subsystem of the WinCE handheld. In our experiments, diagnostic reading of streamed videos at $15 \mathrm{fps}$ was found possible, although 
the evaluation of valve function was sometimes challenging. This justifies the need to overcome the limitation in the frame rate. Further research in various software solutions from vendors indicated that it is technically possible to play videos on WinCE platform with frame rate greater than $15 \mathrm{fps}$. In fact, using Pocket TV MPEG in our lab, we were able to play a previously recorded video of echocardiographic examination at 24 fps. Further research and testing are required in order to extend the limits of the currently available hardware/software solution.

\section{Discussion}

The encouraging results obtained in our initial wireless testbed provide information for planning an improved and more comprehensive wireless infrastructure. We may overcome the limitation due to the dual standard by installing several routers with different pass phrases. This will enable us to selectively enable/disable access of wireless devices to selected routers, thereby improving overall system performance, a prerequisite for our goal of wireless transmission of DVD-quality video (640x480 @ 30fps).

Additionally, we will include simultaneous digital video channels in our wireless network supported by a dedicated media server and web server to provide a user interface to the each channel. To test the multi-channel solution, we will use the existing analog infrastructure currently able to deploy analog video signals from many locations to one specific location within our echocardiography laboratory. We will seamlessly integrate deployment of digital streams into a web based user interface accessible from multi-platform devices (Win CE, Windows, etc).

\section{Conclusions}

While a major strength of ultrasound is its portability, wired network connectivity creates limitations on effective data management. The growth of handheld echocardiographic devices further emphasizes the need for wireless connectivity beyond the boundaries of the hospital.

\section{Acknowledgement}

This study was supported by grants from the National Aeronautics and Space Administration (NCC9-58 and NCC9-60) and the Department of Defense ().

\section{References}

[1] Thomas JD, Greenberg NL, Garcia MJ. Digital Echocardiography 2002: Now is the Time. J Am Soc Echo 2002.

[2] Feigenbaum H. Digital echocardiography. [Review] [19 refs] Am J Cardiol 2000;86(4A):2G-3G.

Address for correspondence.

Neil L. Greenberg, Ph.D.

Department of Cardiovascular Medicine

Cleveland Clinic Foundation, Desk F-15

9500 Euclid Avenue

Cleveland, Ohio 44195 USA

E-mail: greenbn@ccf.org 